\title{
Slip band characteristics in the presence of grain boundaries in nickel-based superalloy
}

DOI:

10.1016/j.actamat.2020.04.037

\section{Document Version}

Accepted author manuscript

Link to publication record in Manchester Research Explorer

\section{Citation for published version (APA):}

Sperry, R., Fullwood, D. T., Harte, A., Fonseca, J. Q. D., Homer, E. R., \& Wagoner, R. H. (2020). Slip band characteristics in the presence of grain boundaries in nickel-based superalloy. Acta Materialia.

https://doi.org/10.1016/j.actamat.2020.04.037

\section{Published in:}

Acta Materialia

\section{Citing this paper}

Please note that where the full-text provided on Manchester Research Explorer is the Author Accepted Manuscript or Proof version this may differ from the final Published version. If citing, it is advised that you check and use the publisher's definitive version.

\section{General rights}

Copyright and moral rights for the publications made accessible in the Research Explorer are retained by the authors and/or other copyright owners and it is a condition of accessing publications that users recognise and abide by the legal requirements associated with these rights.

\section{Takedown policy}

If you believe that this document breaches copyright please refer to the University of Manchester's Takedown Procedures [http://man.ac.uk/04Y6Bo] or contact uml.scholarlycommunications@manchester.ac.uk providing relevant details, so we can investigate your claim.

\section{OPEN ACCESS}




\title{
Slip band characteristics in the presence of grain boundaries in nickel-based superalloy
}

\author{
Ryan Sperry ${ }^{1}$, Allan Harte ${ }^{2,3}$, Joao Quinta da Fonseca², \\ Eric R Homer ${ }^{1}$, Robert H Wagoner ${ }^{4}$, David T Fullwood ${ }^{1}$ \\ ${ }^{1}$ Brigham Young University, Provo, Utah, USA \\ 2 The University of Manchester, UK \\ ${ }^{3}$ UKAEA, Culham Science Centre, Abingdon, Oxon, OX14 3EB, \\ ${ }^{4}$ The Ohio State University, Columbus, Ohio, USA
}

\begin{abstract}
Shear strain profiles along slip bands in a modified Rolls-Royce nickel superalloy (RR1000) were analyzed for a tensile sample deformed by $2 \%$. The strain increased with distance away from a grain boundary (GB), with maximum shear strain towards the center of the grain, indicating that dislocation nucleation generally occurred in the grain interior. The strain gradients in the neighborhood of the GBs were quantified and generally correlated with rotation about the active slip system line direction. This leads to an ability to determine the active slip system in these regions. The dislocation spacing and pileup stresses were inferred. The dislocation spacing closely follows an Eshelby analytical solution for a single ended pileup of dislocations under an applied stress. The distribution of pileup stress values for GBs of a given misorientation angle follows a log-normal distribution, with no correlation between the pileup stress and the GB misorientation angle. Furthermore, there is no observed correlation between various transmissivity factors and slip band pileup stress. Hence it appears that the obstacle strength of any of the observed GBs is adequate to facilitate the dislocation pileups present in the slip bands. However, slip band transmission does correlate with transmissivity factors, with the current study focusing on the Luster and Morris $\mathrm{m}^{\prime}$-factor. Observation of strain profiles of transmitted bands indicate dislocation nucleation locations.
\end{abstract}

Keywords: Slip Band, slip transmission, dislocation theory, digital image correlation, electron backscatter diffraction (EBSD)

\section{Introduction}

Despite the known importance of dislocation / grain boundary (GB) interactions, most mesoscale models do not account for these interactions at the slip system level. A large factor in this scientific gap is the lack of detailed observations of dislocation / GB interactions across the wide range of GB types. Description of these GB types include several characterizations of the interface between two discrete grains. These include characterizations of the GB itself such as GB tilt etc. [1-5], as well as characteristics of the grains on either side of the GB such as relative misorientation etc. [6-8]. The latter will be used for this paper in observing dislocation / GB interactions and will be referred to as the GB character as it characterizes the grain-grain interface. While the observation of single dislocation behavior in the presence of GBs is difficult 
to observe for a statistically significant number of cases, slip-band interactions can be observed much more readily using various techniques.

This paper employs high-resolution digital image correlation (HRDIC), combined with electron backscatter diffraction (EBSD) to study slip band interactions with GBs for several hundred bands, across a wide range of GBs. The source and nature of the underlying slip activity, the associated geometrically necessary dislocation (GND) structure and resultant stress concentrations, as well as the transmission behavior across GBs is investigated.

Slip bands are the dominant mechanism for plastic deformation in a range of polycrystalline materials. For face-centered cubic (FCC) nickel-based superalloys, there are generally one or more favorably oriented slip systems for accommodation of strain within each grain, often resulting in a single dominant system of parallel slip bands across the grain $[9,10]$. However, the behavior of the bands as they approach grain boundaries (GBs) can be more varied, with some bands crossing, and others fading and terminating or breaking up as they approach the obstacle $[11,12]$. While some believe the GB to often be the dominant source of dislocations that compose slip bands [13], there is still significant uncertainty in the literature as to where these dominant sources lie. This paper will investigate possible evidence of sources being elsewhere in the grain and thus build upon current understanding of dislocation / GB interactions.

In the classical view of the slip band / GB interaction, proposed by Hall and Petch [14,15], dislocations that form in the grain interior glide until they encounter a GB that prevents further motion and pins the leading dislocation [16]- this motion prevention is influenced by the level of difficulty for slip to occur in the adjacent grain; dislocations following behind a pinned dislocation are repelled by the elastic dislocation-dislocation forces, and a pileup occurs (see Li and Chou for a discussion of this scenario, and alternative hypotheses [17]). Of particular importance in this view is the GB obstacle strength or the stress required to push the dislocation into or through the boundary. This slip "transmission" can also be considered activation of slip in the neighboring grain. However, it will be referred to as transmission in this paper as it is the term often used in the literature to reference this phenomenon $[1,2,5,18]$. Hence the fundamental question arises as to whether the observed pileup and / or the transmission (as described by pushing dislocations through a GB as well as activation of slip in neighbor grain) behavior at GBs correlates with GB obstacle strength.

Measured or calculated values of obstacle strength are not available for most GBs, in any material; some inferred values exist for a limited number of observations (e.g. [16]), and various analyses of certain GB subsets have been attempted with molecular dynamics (e.g. [18]). Nevertheless, there is a generally accepted notion that the obstacle strength will correlate with geometrical transmissivity factors [19] that quantify the misalignment of incoming and outgoing slip systems. Thus, the current paper investigates the existence of correlations between these transmissivity factors and both the observed pileup stresses and observed transmission behavior. 


\subsection{Slip band identification}

A polycrystalline nickel superalloy specimen was investigated: RR1000 with $\gamma^{\prime}$ precipitate which is a material that was developed at Rolls Royce plc. It has a preferred composition by weight percent of 14.0-19.0\% cobalt, 14.35-15.15\% Chromium, 4.25-5.25\% Molybdenum, 1.35-2.15\% tantalum, 3.45-4.15\% titanium, 2.85-3.15\% aluminum, 0.01-0.025\% boron, 0.012-0.033\% carbon, $0.05-0.07 \%$ zirconium, $0.5-1.0 \%$ hafnium, up to $1.0 \%$ rhenium, up to $2.0 \%$ tungsten, less than $0.5 \%$ niobium, up to $0.1 \%$ yttrium, up to $0.1 \%$ vanadium, up to $1.0 \%$ iron, up to $0.2 \%$ silicon, up to $0.15 \%$ manganese and balance nickel plus incidental impurities $[20,21]$.The $\gamma / \gamma^{\prime}$ alloy was subjected to heat treatment in order to arrive at unimodal size distributions of $\gamma^{\prime}$ precipitates with diameters of $\sim 70 \mathrm{~nm}$. To do this, a $\gamma^{\prime}$ super-solvus solution heat treatment was applied, then the sample was quenched with oil and followed by a $\gamma^{\prime}$ super-solvus solution heat treatment and very slow cooling to promote grain growth rather than nucleation of new grains. Details of the heat treatment are given in [22]. The average grain size (equivalent circle diameter) for the alloy was approximately $43 \mu \mathrm{m}$ weighted by area. As will be noted again later, the misorientation distribution follows the trend noted by Mackenzie [23]

A tensile specimen was machined and polished for pre-deformation EBSD orientation mapping using standard mechanical polishing processes [22]. Then, a gold speckle pattern was applied to the sample surface for the image correlation process, following the procedure detailed in [11]. Images of the speckle pattern were obtained before and after uniaxial tensile deformation, in the unloaded state, and the resulting displacement field was calculated using LaVision's commercially available DIC software, DaVis 8 [24]; software parameters were selected to provide an error of below 0.01 effective strain for the approximately 32 million data points [25].

Backscattered electron images of the gold speckle pattern were obtained before and after the deformation step using a FEI Magellan HR 400L FE-SEM. To maximise the spatial resolution, the microscope was operated at a voltage of $5 \mathrm{kV}$ with a $+2 \mathrm{kV}$ stage bias and a probe current of 0.8 $\mathrm{nA}$. A working distance of $4 \mathrm{~mm}$ was chosen to maximise the signal-to-noise ratio. A mosaic of 40 columns $\times 20$ rows was used to collect 800 images with a $20 \%$ overlap, corresponding to a field of view of $\sim 1 \times 0.5 \mathrm{~mm}$. Each image had a resolution of $2048 \times 1768$ pixels and a pixel size of $14.6 \mathrm{~nm}$.

The dogbone sample was deformed in uniaxial tension using a Zeiss-Kammrath $5 \mathrm{kN}$ tensioncompression microtester. The sample was deformed at a rate of $0.3 \mathrm{~mm} \mathrm{~min}^{-1}$ to a global macroscopic uniaxial engineering strain of $\sim 0.02$. 


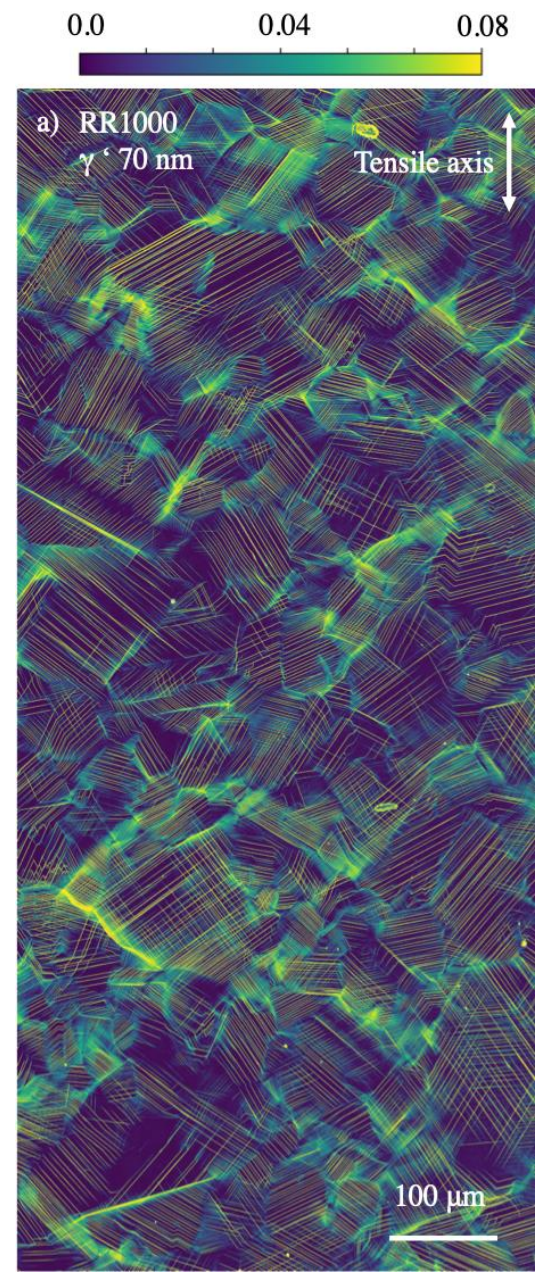

Figure 1. Maximum shear maps from HRDIC analysis of RR1000 with precipitates.
The locally calculated values of displacement, $u$ and $v$, from DaVis, were used to determine the maximum shear strain, under a 2D plane strain assumption [26]:

$\frac{\gamma_{\max }}{2}=\sqrt{\left(\left(\frac{\partial u}{\partial x}-\frac{\partial v}{\partial y}\right) / 2\right)^{2}+\left(\left(\frac{\partial u}{\partial y}+\frac{\partial v}{\partial x}\right) / 2\right)^{2}}$

Where $\gamma_{\text {max }}$ is the shear strain and $\frac{\partial u}{\partial x}, \frac{\partial v}{\partial y}, \frac{\partial u}{\partial y}, \frac{\partial v}{\partial x}$ are the displacement gradients in the $x$ and $y$ directions. Note that the level of shear across a slip band was measured relative to the step size of the DIC grid $(117 \mathrm{~nm}$ for the DIC window size of $8 \times 8$ pixels) and does not represent the actual shear based upon the distance between neighboring slip planes; the calculated value provides somewhat of a lower bound of shear. This does not affect the calculations of dislocation spacing below, which are based upon the displacements across the slip plane. The resultant strain maps have sharp bands of high shear strain values along the slip bands, as illustrated in Figure 1. These sharp bands will be measured as discussed later. Important to note is that if slip is occurring in a direction nearly parallel with the surface, a slip trace would not be seen in the maximum shear map. However, this wouldn't influence the analysis that will be done on each slip band.

The HRDIC map was spatially registered with the maps of EBSD data by manually selecting a series of triple junctions in both maps and minimizing the least square error between these keypoint positions while applying a linear transformation to the EBSD map. We note that this does not give perfect alignment, with grain boundary positions in the two maps typically being displaced from each other by a few microns. Grains in the DIC map were defined using the EBSD data and were analyzed individually for slip band activity.

The strain map for the grain was passed through a Radon transform, and the peak values indicated the slip band locations. Shear strain vs distance from the GB along the band was extracted from the HRDIC shear strain map. Because of the small error between GB location as defined by the overlaid EBSD map, and the actual GB location in the DIC map, the ends of the slip bands were generally defined by the point of minimum shear value rather than the approximated GB position, significantly mitigating the slight misalignment issue. Spacing between bands were measured by taking perpendicular lines from the center of each identified band, mapping the shear strain along this line, and finding the distance to the first peak, indicating the distance to the neighboring band. This was performed automatically using the 
"findpeaks" function in MATLAB, but was also checked manually over a significant number of bands.

Each straight and clear slip band was automatically identified, and then manually checked by examining the proposed endpoint positions on the shear and EBSD maps, as well as the associated shear profiles along the bands. This was done for each band to confirm the automated program had successfully selected a slip band. We also note that shear bands that are not straight (e.g. that zig-zag across the grain), or that are particularly weak, would not be identified by the Radon transform method. Please refer to Figure 1 or Figure 4 for visualization of the sharp and straight bands identified as well as zig-zag or weak bands that are not identified. After the automatic identification and manual check process was completed, 660 bands were available for examination.

For each slip band, perpendicular lines were defined, and the relative displacement ratio (RDR), $\left(u_{2}-u_{1}\right) /\left(v_{2}-v_{1}\right)$, across the band was determined, as described by Chen and Daly [27]; $u_{1}$ and $u_{2}$ represent displacements of two points on either side of the shear band in the global xdirection, and similarly for the y-direction displacements represented by $v_{i}$. For the known crystal orientation (measured by EBSD), potential slip planes whose traces aligned closely with the observed slip band were determined. The measured RDR was then compared with the possible RDRs relating to the identified slip plane(s). Thus, the active slip system associated with the selected band was determined. Once this was known, the component of displacement perpendicular to the sample surface could be determined from the measured $\mathrm{x}$ and $\mathrm{y}$ displacements (assuming that only one of the three possible slip directions in a slip band was active, which is the required assumption for this RDR method). Chen and Daly did validation studies on slip systems identified with high confidence through other methods $[27,28]$. Their method proved to match well with predictions from these validation studies. Shear and displacement values were then modified by resolving the two-dimensional values calculated from the displacement field into the three-dimensional slip direction as determined from the RDR method discussed.

\subsection{The Frank-Read source and dislocation loops}

The relative displacement along slip bands indicates the number of dislocations that have passed along the slip plane; e.g. for a slip plane that lies in the $x-z$ plane, with an edge dislocation line parallel to the $z$-axis and with a slip direction along the $x$-axis, the total relative displacement along the slip band will be $n b$, where $n$ is the number of dislocations that have passed a given point, and $b$ is the size of the Burgers vector. To create the observed sharp slip bands shown in the HRDIC scans, there must be consistent successive generation of dislocations on the slip band. This effect can be attributed to Frank-Read sources [29] which are generally caused by applied stress to pinned dislocation line segments. Note that this method assumes that slip for a slip band occurs along a single plane while it is understood that slip bands are 
composed of slip on several very closely spaced glide planes [30]. However, assumption of slip on one plane is adequate for the displacement dislocation relationship mentioned above as the total displacement over the width of the band is summed up and thus incorporating all dislocations in the slip band as a superdislocation. As this paper discusses slip on a single plane, it is referring to the mathematical assumption through the superdislocation summation model.

In the presence of a barrier to dislocation glide, such as a grain boundary, a dislocation pileup of successive Frank-Read dislocation loops may occur against the locked dislocation that is closest to the barrier. In this case, there is a strain gradient in the direction of the pileup. If all dislocations originated at the center of the grain (consistent with observations detailed below), and if the spacing of the dislocations at a certain value of $x$ is $w$ with the Burgers vector magnitude equal to $b$, then the displacement gradient, $\frac{d u}{d x}$, and strain gradient, $\frac{d \varepsilon}{d x}$, at that point (on the side of the slip plane with the dislocation motion) will be given by:

$$
\frac{d u}{d x}=\frac{b}{w} ; \frac{d \varepsilon}{d x}=\frac{-b}{w^{2}} \frac{d w}{d x}
$$

The resultant dislocation spacing and strain gradients can be compared with Eshelby's analytical solutions for the distribution of dislocations in pileups. We consider 1) A double ended pileup between two pinned dislocations, with no externally applied stress, and 2) A single directional pileup against a pinned dislocation, under evenly applied stress [31]. The solution for dislocation positions in the first case is given by the roots of the first derivative of the Legendre functions given by:

$$
f=P_{n-1}^{\prime}\left(\frac{x}{L}\right)
$$

where $L$ is equal to the length of the slip band and $x$ is equal to the position along the slip band and represents a set of more evenly distributed dislocations compared with the second case, where the pileup is more severe near the pinned dislocation; the positions of dislocations for this case are given by the roots of the first derivative of the Laguerre functions given by:

$$
f=L_{n}^{\prime}\left(\frac{2 \tau_{0} x}{A}\right)
$$

where $\tau_{0}$ is equal to the applied shear stress, $A$ is equal to the slip area, and $x$ is equal to the position along the slip band. For the purpose of this paper, $\tau_{0}, A$, and $L$. Are not needed as normalized positions of dislocations will be used to create a normalized theoretical shear strain curve for the corresponding pileups. 


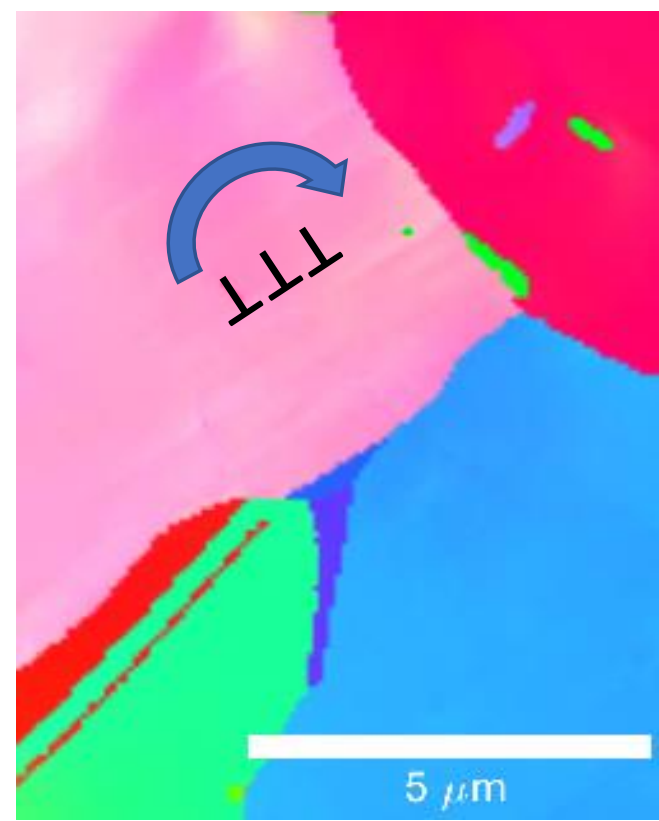

Figure 2. Illustration of the lattice rotation about the dislocation line direction in a pileup.
A further characterization of GND distributions towards the ends of slip bands was carried out by analyzing the orientation gradient field in the vicinity of the band. In the scenario of a series of edge dislocations aligned along the slip plane, in the slip direction, the crystal lattice should rotate about the dislocation line direction, as shown in Figure 2, by an amount that relates directly to the dislocation density. For $n$ edge dislocations in the step $\Delta x$ along the slip direction, the lattice curvature $K$ is given by [32]:

$$
K=\frac{d \theta}{d x}=\frac{d \gamma}{d x}=\rho b
$$

Where dislocation density $\rho=1 /(w h)$ and $w=$ $\frac{\Delta x}{n}$, giving the spacing between the dislocations in the band, and a spacing $h$ between bands; $b$ equals the magnitude of the Burgers vector. The measured axis of rotation can then be compared

to the line direction of the slip band evaluated using the RDR method.

Furthermore, the direction of the rotation would suggest which portion of the dislocation loop is observed at the surface. Dislocation loops are composed of edge dislocations of opposite signs at either end of the loop (top and bottom of the loop in Figure 3 ) with screw dislocations between the edge dislocations [33]. The relative signs of observed edge dislocation pileups will depend on which portion of the loop relative to the Burgers vector direction is observed at the surface. Furthermore, if edge dislocation pileups are observed at both ends of a slip band, the relative signs of the edge dislocations can be determined from the local rotation gradients near the grain boundary. When the rotation direction at each end of the band is calculated using the center of the band as a reference point, rotations of the same direction at each end would suggest edge dislocations of opposite signs. Rotations of the opposite direction would suggest edge dislocations of the same sign in both of the pileups at each end. It is important to also note that depending on the direction

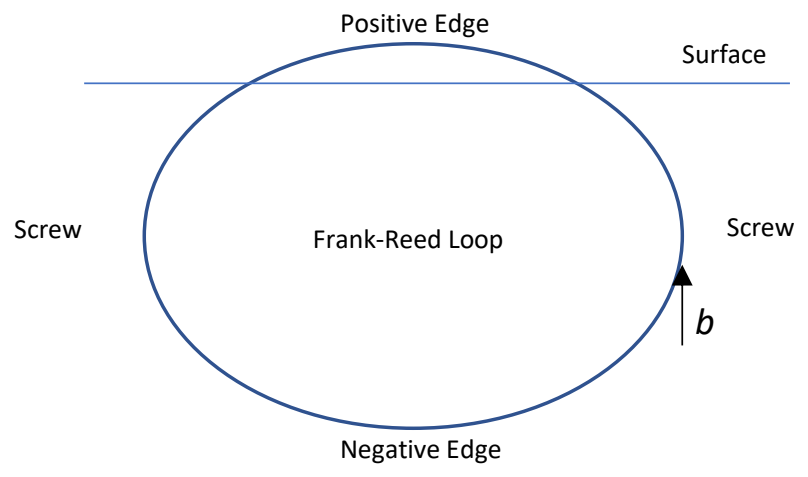

Figure 3. Simple diagram of Frank-Read loop interacting with the sample surface showing edge dislocations of the same sign at the surface. If the Burgers vector was rotated 90 degrees such that the screw dislocation portion is pushed entirely out of the sample surface, the dislocations observed at the surface would be of opposite sign. 
of slip and development of the dislocation loop, there may be screw dislocation pileups which may affect the axis of rotation. This will be revisited later as the results are being discussed and is intended to be part of the focus of another study.

\subsection{Analysis of Pileups}

Of particular interest to the current study is the stress applied to the grain boundary by dislocation pileups within a slip band, and any observed correlations between this stress and the GB character. Eshelby showed that for a pileup caused by an applied stress, $\tau$, the stress on the pinned dislocation would be given by $N \tau$, where $N$ is the number of dislocations in the pileup. For the case of the pileups observed in the nickel superalloy grains, with unknown residual stress after unloading the sample, the pileup stress might be approximated by fitting Eshelby's solutions (such as those mentioned above) to the observed strain gradient and backing out the associated stress. However, a more direct approach involves analysis of the dislocation spacing that is required to produce the observed strain gradient, and then integration of the elastic forces applied by such a dislocation field upon the pinned dislocation. For edge dislocations moving in the same slip plane, the applied stress is summed over a set of integration steps with $n_{i}$ dislocations in the given step (derived from $w$ in Eq. 2), at a distance $x_{i}$ from the pinned dislocation [34,35]:

$$
\sigma_{x y}=\sum \frac{\mu b n_{i}}{4 \pi(1-v)} \frac{l}{x_{i} \sqrt{x_{i}^{2}+(l / 2)^{2}}}
$$

where $\mu$ is the shear modulus, $b$ is the Burgers vector, $v$ is the Poisson's ratio, and $l$ is the length of the dislocation - taken to be the visible grain diameter in this work. The shear modulus and Poisson's ratio are $75 \mathrm{GPa}$ and 0.3 respectively for the nickel superalloy material while $l$ varies between bands in different grains. We note that the grain diameter estimate will be a source of variability in the results; but since the stress contribution is higher for small $x_{i}$, when the $l$ terms approximately cancel, this should not cause a large error. Additionally, as will be seen, the stress calculation is important for relative trends and comparisons, thus the exact value is not as important to this study. Any error in the $l$ terms would cause a scaled increase/decrease to all stress values calculated under the same assumptions for each slip band and give the same relative results. Another important note with this equation is that it is under the assumption of a superdisocation model with all dislocations summed across the width of the slip band and assumed to be on one plane as discussed previously. Since in reality dislocations are on several parallel slip planes, the actual backstress would be slightly less than what is calculated using this equation. This overestimation would be relatively insignificant as lateral variation of dislocation position would be of orders of magnitude lower than variation of dislocation position along the band. Nevertheless, this equation can be used as an upper limit and since slip band thickening is consistent among slip bands, relative trends should still be the same. 
Since the total force is most affected by the dislocations closest to the pinned dislocation, the integral of force is sensitive to step sizes used on data close to the pinned dislocation. To maximize computation efficiency and speed, a smaller step size equal to the magnitude of the Burgers vector of one full edge dislocation in nickel (approximately .249 nm) was used for the first three microns of each shear band. The rest of the band used a step size of .05 microns. It was found that using this step size beyond the first three microns had an insignificant effect on the calculated stress when compared to using a step size of the Burgers vector for the entire shear band. The data from the DIC maps were interpolated to these refined step sizes, and locally smoothed to reduce numerical noise.

It should be noted that the data was extracted in the unloaded conditions, and hence does not indicate the maximum stress that was applied to the GB by the shear band under load. But it is assumed that the unloaded strain will be proportional in some way to the loaded value and insitu tests have shown that any change in plastic strain within the bands during unloading is too small to be resolved with the HRDIC technique [36].

\subsection{Transmissivity Factors and Transmission}

As previously noted, one objective of the current study is to determine correlations between pileup stress exerted on GBs, and microstructural metrics that might relate to the obstacle stress of the given boundaries. Metrics that have arisen in the literature include GB misorientation and the Luster and Morris $\mathrm{m}^{\prime}$-factor, as given by [6]:

$$
m^{\prime}=\max _{\alpha}\left\{\hat{n}_{s b} \cdot \hat{n}_{\alpha} * \hat{b}_{s b} \cdot \hat{b}_{\alpha}\right\}
$$

where $\hat{n}_{s b}$ and $\hat{b}_{s b}$ are unit vectors perpendicular to the slip plane and in the direction of slip, respectively for the shear band being examined; and $\hat{n}_{\alpha}$ and $\hat{b}_{\alpha}$ are unit vectors for all slip systems in the neighboring grain.

Other factors such as the Chalmers N-Factor [8], the Lambda parameter from Werner and Prantl [7], and the residual Burgers vector (RBV) [37] were used to measure transmissivity. However, as will be seen below, this study focused on $\mathrm{m}^{\prime}$ and GB misorientation because they are common metrics in the literature and showed the same results as all the other transmissivity factors mentioned with regards to grain boundary backstress.

Higher values of metrics $m^{\prime}, N$ and $\lambda$ indicate better aligned slip systems across the $\mathrm{GB}$, and hence easier transmission. For the RBV metric, a high value indicates that for a dislocation to transmit through the $\mathrm{GB}$, if the net Burgers vector is conserved, a high residual Burgers vector must be retained in the $\mathrm{GB}$, presumably at a high energetic cost, thus correlating with more difficult transmission. 
Other microstructural attributes that may correlate with transmissivity include the misorientation of the GB of interest, along with other metrics such as combinations of Schmid or Taylor factors of the neighboring grains.

\subsection{Transmission}

As mentioned previously, one type of interaction that can be readily observed between slip bands and GBs is transmission events. If a slip band is transmitting through a GB, then the HRDIC scans should be able to pick up on the continuous shear through the GB caused by the transmitted dislocations. We note that if a transmission event nucleated below the surface, there may be no reason for the slip bands on either side of the GB to line up at the surface. However, there is some evidence to indicate that transmission events primarily nucleate at the free surface, when such a surface is present [38] which will be discussed further below. The character of strain and strain gradients in slip bands at grain boundaries, for both visibly transmitting and non-transmitting bands, was catalogued to identify a useful classifier for automatically determining if a band transmitted or not. The bands classified as transmitting bands were then compared with transmissivity factors mentioned above to see if there were any correlations between transmission and these factors.

The shear profiles of transmitted bands were also analyzed to obtain insight on the primary source of dislocations for the bands in both non-transmitting and transmitting cases. Since shear is proportional to the number of dislocations that have passed along a slip band, local maxima, minima, and gradients of the shear profiles can be useful indicators of the source and evolution of the dislocations in these bands.

\section{Results and Discussion}

Using the automated method described above 660 bands were identified in the RR1000 material. Note that modifying the parameters of the Radon transform search method would alter the number of bands that are found in the material. A typical plot showing the distance along a slip band vs maximum calculated shear strain can be seen in Figure 4 . Note that the maximum shear is near the grain center and the minimums are at the GB's. As discussed before, shear strain calculated across a single pixel width band is proportional to the amount of displacement at a particular point. Displacement at a particular point is caused by dislocations passing through the specified point, so shear strain values correlate with the number of dislocations that have passed through the specified point. As dislocation loops propagate outward from their sources and continue gliding until they get pinned or stop, a peak in shear along a slip band would suggest that most dislocations are being generated at this location because all would pass through locations immediately adjacent to the dislocation source, and as they stop or get pinned moving away from the source the shear values would decrease 
creating a negative gradient on either side. According to the peak in Figure 4, the source of the majority of the dislocations in a slip band is close to the center of the grain where the the maximum value of shear strain is found. The majority of shear bands showed similar behavior. One thing to note, however, is that it is possible to have multiple dislocation sources along the slip band, and still have the final distribution of the dislocations creates a shear profile that would be the same as if there was a dominant dislocation source in the middle of the grain.

Figure 5 presents a boxplot of the normalized shear strain data along the 660 slip bands in the RR1000 material. The shear profile for each band is normalized to a maximum value of 1 (after
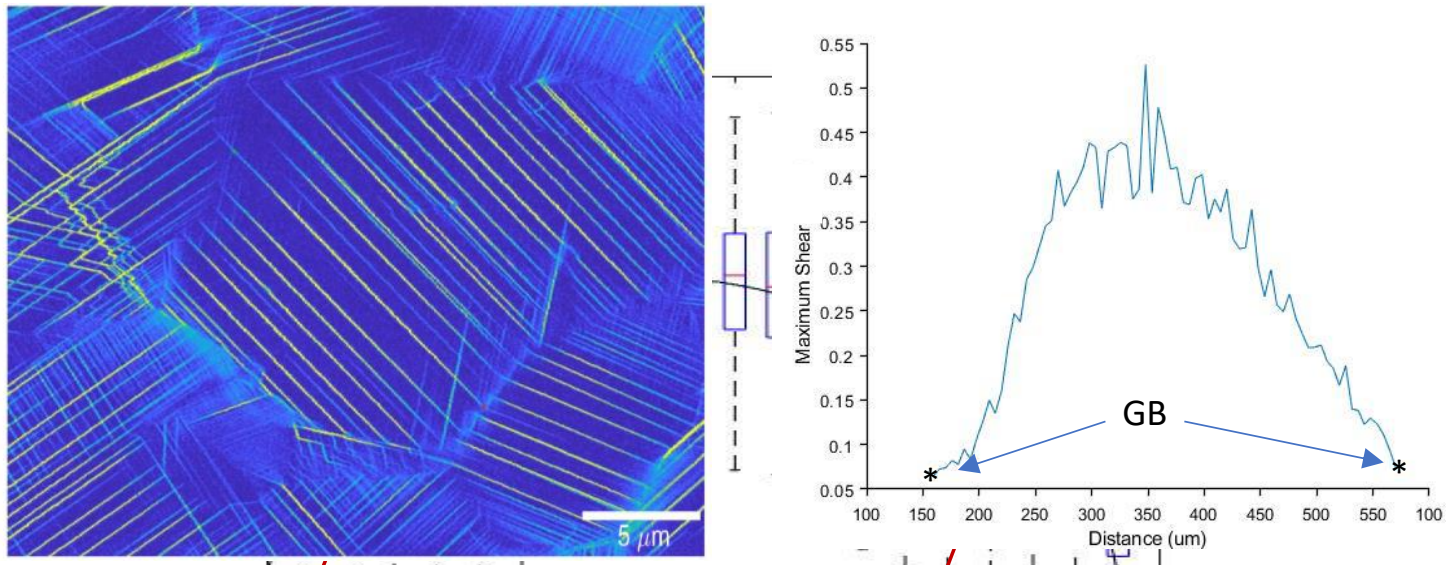

Figure 4. Typical map of maximum shear strain from HRDIC scanning with visible slip bands (left) and typical shear profile of maximum shear strain vs. distance along the band starting at one $G B$ and moving toward the other (right). GB is indicated by the asterisks.

Figure 5. Box plot showing the normalized shape of shear profiles across 660 slip bands in RR1000 fine precipitate material (the middle lines show the median, the box shows 25th-75th percentile values, and the dashed lines show the extent of the other non-outlier values); solutions from two Eshelby pileup scenarios overlaid on the box pot.

being resolved into the slip direction as mentioned above), and a length of 20 arbitrary units (a.u). Starting at the GB at one end of a slip band, the median profile of shear increases for the first third of the slip band, levels out for the middle third, and then decreases for the final third of its length, as the opposite GB is approached. As mentioned earlier, this was compared to the solutions proposed by Eshelby for shear strain in dislocation pileups. Since the sample is in the unloaded state, one might assume that the first Eshelby solution (dislocation pileup between two pinned dislocations with no applied stress; dislocation positions correspond with zeros of the first derivative of a Legendre polynomial [31]) would be more relevant; however, the second Eshelby solution actually appears to be a significantly better fit to the data (dislocation positions correspond with the zeros of the first derivative of a Laguerre polynomial [31]). Hence, it appears that after the sample is unloaded, the dislocations in the slip band do not fully reverse to take the spacing of the ideally unloaded pileup. This final unloaded dislocation arrangement leads to long-range backstresses [34]. 
As mentioned earlier, the strain gradients caused by these dislocation pileups correlate with orientation gradients in the material. In the RR1000 material, 20 bands were selected by randomly choosing 10 grains and then randomly selecting 2 bands per grain. These bands were used to evaluate these orientation gradients. It was found that $85 \%$ (17 of the 20) of the selected bands showed rotation about an axis that matched up with the line direction as identified using the RDR method. This gives strong supporting evidence that the active slip system can be identified through rotation caused by localized lattice strain from dislocation pileups. However, it must be noted that it may be necessary to have similar global strain levels and manifestations of sharp and clear slip bands. This finding enables the use of lattice rotation measurement methods such as EBSD scanning to be used for active slip system identification rather than costly HRDIC scanning. While these findings are quite promising, this is planned to be the topic of a future study for further evaluation of the method's efficacy with a more detailed analysis.

Furthermore, the direction of the rotation suggested that 11 of the bands had edge dislocations of similar sign on either end of the slip band while 6 bands suggested edge dislocations of opposite sign as shown in Figure 6. One possible explanation to the bands that didn't fit either case could be caused by the dislocation pileups being associated with screw dislocations, such as when the burgers vector is close to perpendicular with the surface (refer to Figure 3 ), or mixed dislocations from the dislocation loop. This would potentially cause unexpected lattice

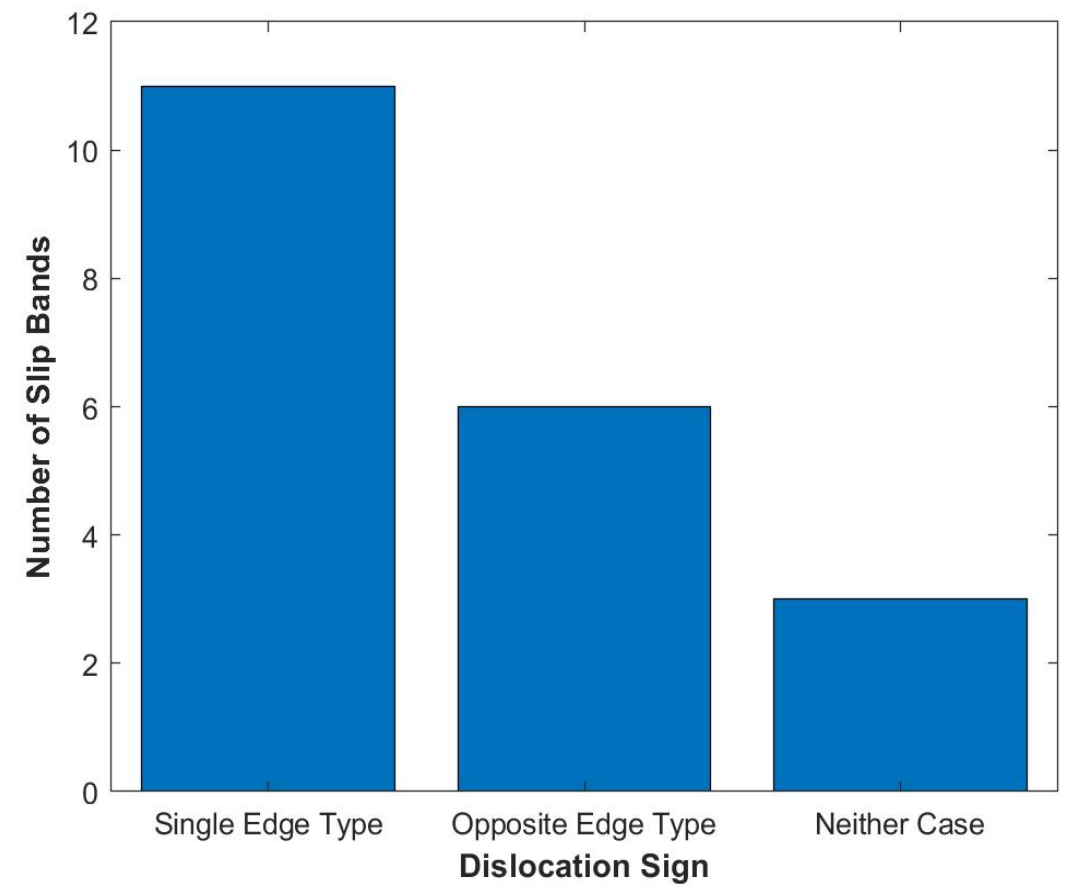

Figure 6. Graph of number of slip bands with single edge dislocation type and opposite edge dislocation type at each end of the band as observed through lattice rotation. 
rotation and is intended to be an area for further study. This shows supporting evidence that the slip bands observed in the material fit the Frank-Read source dislocation formation model.

We now consider correlations between the pileup stress calculated in the region of GBs, and microstructure characteristics. Data for the relationship between pileup stress and GB misorientation is shown in Figure 7 which, as mentioned earlier, is representative of all transmissivity factors calculated, since the trends were virtually identical. This representation will also be visited again later in the paper. One can see that the number of data points also increases with misorientation, then declines; with a final spike in data points at $60^{\circ}$. The $60^{\circ}$ GBs represent twin GBs, with approximately $1 / 3$ of the $G B s$ being associated with twins. The distribution of non-twin GB types closely follows the trend noted by Mackenzie [23] for GB

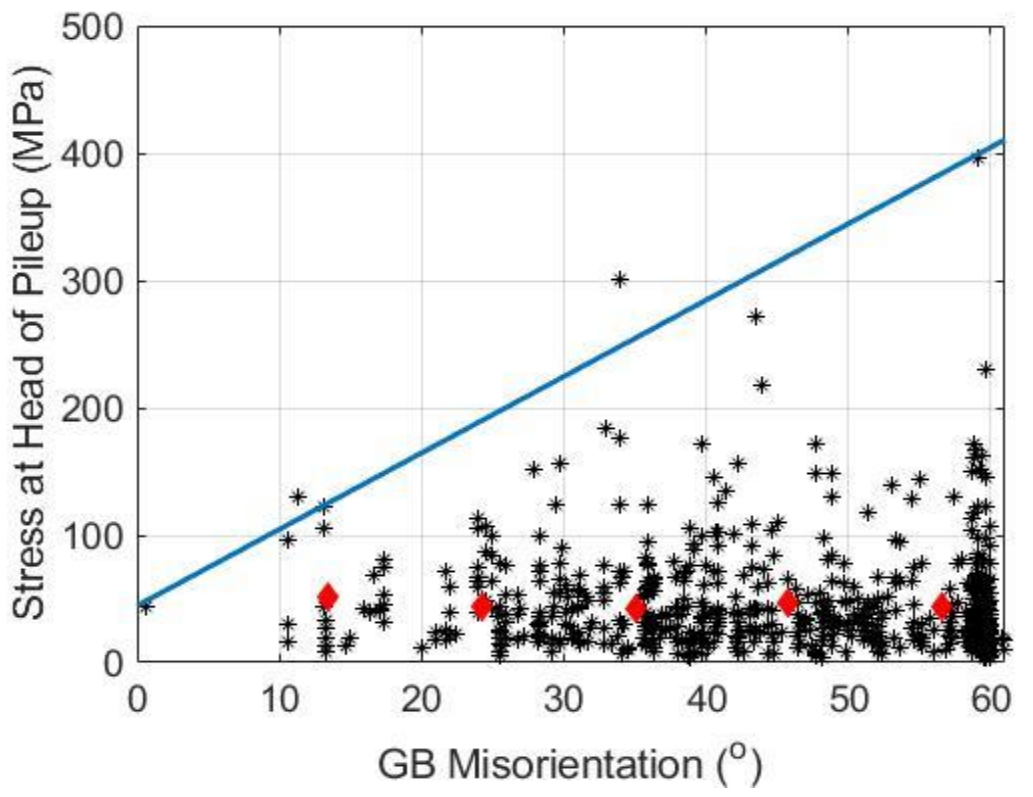

Figure 7. Misorientation vs stress at head of GB pileup. Red diamonds indicate mean stress. Blue line indicates upper bound (trendline of maximum stress vs GB misorientation) for data.

misorientation distribution in a random texture, with a peak density at around $40^{\circ}$. If the mean stress is plotted against misorientation (red diamonds), there is no apparent trend; the mean stress is constant with misorientation. It may seem from the figure that the maximum stress (or upper bound of all data points) for a given orientation increases as misorientation increases (blue line). However, this correlation of maximum stress with misorientation appears to simply be a result of an increase in the number of points sampled at a given misorientation, which statistically would include points from further along the tails of the stress distribution and give higher maximum values.

In order to investigate the factors that contributed to the stress distribution at a particular type of GB (in this case, a GB with a given misorientation), the distribution of stress for two different 
types of GB were considered further. The pileup stress at twin GBs (a total of $212 \mathrm{GBs}$ ) and at GBs with misorientation between $35^{\circ}$ and $45^{\circ}(157 \mathrm{GBs})$ was assessed. Figure 8 shows the data for both types of $\mathrm{GB}$, indicating a log normal distribution of pileup stress; this strengthens the

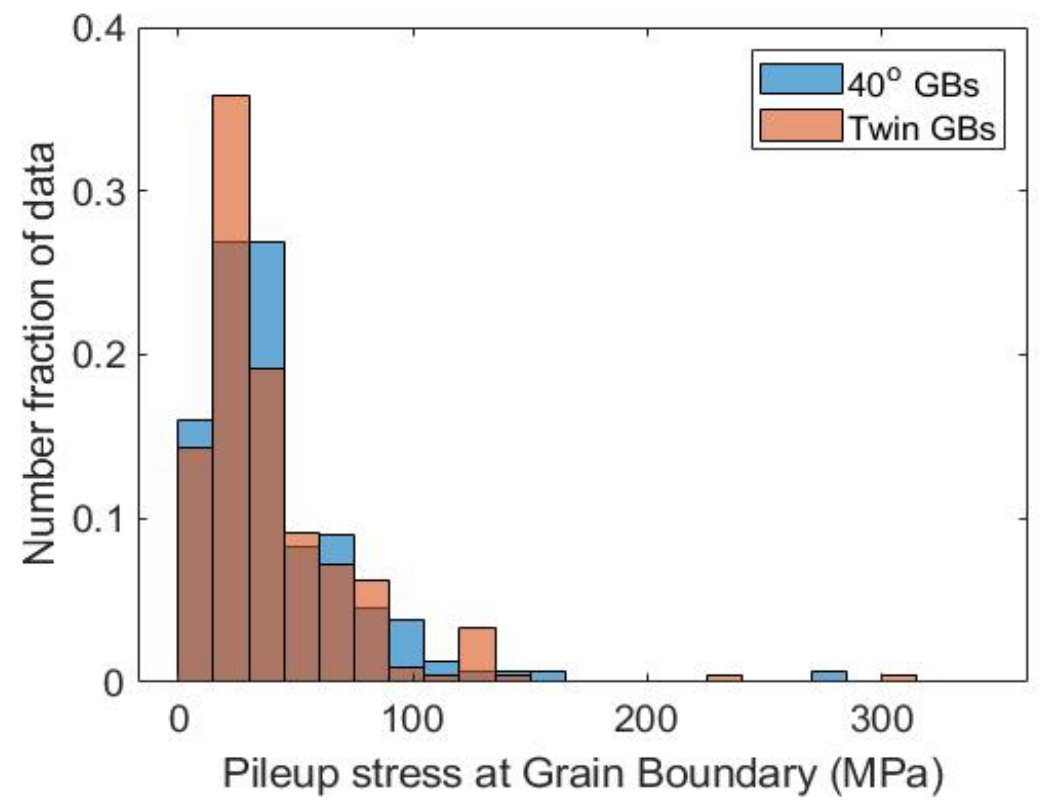

Figure 8. Distribution of pileup stresses observed across 157 GBs with nominal misorientation of 40, and for 212 twin GBs. Note for better visualization of the distribution, the highest twin pileup stress is not shown, but can be seen in fig. 7.

hypothesis that the positive correlation of the upper bound of the data points is most likely simply the result of a higher number of data points being sampled from the distribution.

For the pileup stress relating to a single $\left(\sim 40^{\circ}\right)$ misorientation, various microstructural characteristics were tested for correlation with the level of pileup stress. The strongest correlation related the pileup stress to the maximum shear strain across the relevant shear band. The $p$-value for the statistical relationship between pileup stress and maximum shear strain along the shear band was almost zero (1e-5), indicating a statistically significant correlation between these factors; on the other hand, the $\mathrm{R}^{2}$ value for the correlation was only 0.115 (Figure 9), signifying the likelihood that other factors are important. As pointed out by previous researchers, we note that there was no significant correlation between the Schmid factor of the shear bands that develop within a given grain, and the maximum shear that develops [25].

Importantly, there were no significant correlations between transmissivity factors (the assumed indicators of differing obstacle stress) and pileup stress for a given GB misorientation. Hence the differing pileup stress for a given GB type does not appear to correlate with GB obstacle stress. 
In summary, there is no correlation between pileup stress and transmissivity factors (GB misorientation, Luster-Morris factor, etc.) as figure 7 suggests; the data being representative for all transmissivity factors. The positive correlation between the upper bound of the pileup stress and transmissivity factor (GB misorientation) appears to be caused by the number of sampled points in transmissivity factor value ranges. There is a slight correlation between

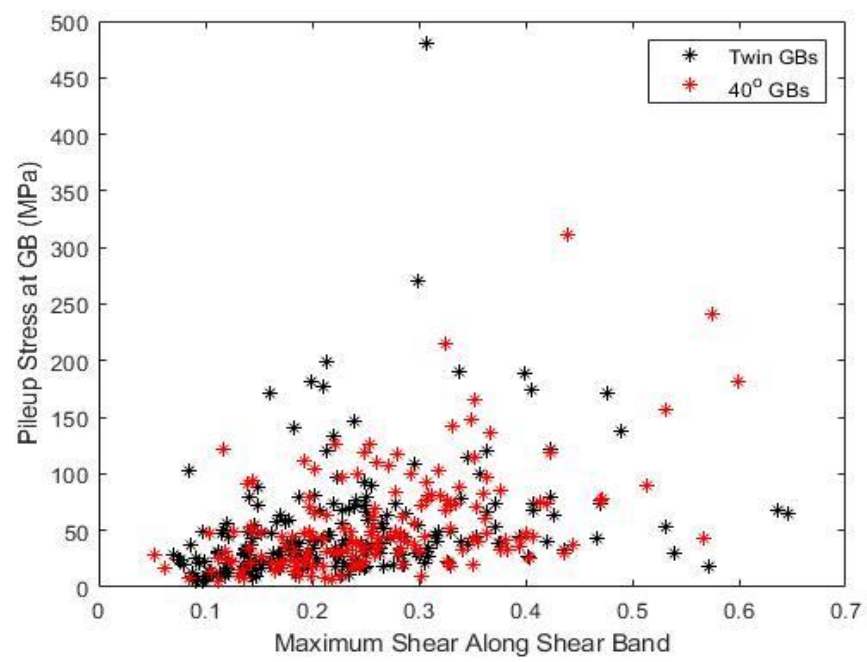

Figure 9. Pileup stress vs maximum shear stress in the band for 157 slip bands with nominally $40^{\circ}$ misorientation, and for 212 twin GBs in the material.

maximum shear strain accumulation within grain and pileup stress for a given GB misorientation, with a relatively weak correlation coefficient that indicates that other factors are also likely to contribute. 
We now look for potential correlations between pileup stress and transmissivity factors across all slip bands in the material, without holding the misorientation constant. The $\mathrm{m}^{\prime}$-factor metric is the simpler of the transmissivity metrics and is similar to the $\mathrm{N}$-factor in terms of definition; the $\mathrm{m}^{\prime}$ and $\mathrm{N}$-factor metrics have a positive linear correlation with R-squared factor of 0.77. Figure 10 illustrates the relationship between transmissivity (as measured by $\mathrm{m}^{\prime}$ ) and GB misorientation. When the misorientation is low the transmissivity is high (i.e. slip systems in neighboring grains are reasonably well aligned); when the misorientation is high the transmissivity generally decreases but can still take relatively high values at high misorientation values for specific relative orientations. The twin GBs appear to take one of three discrete transmissivity values, with approximately $1 / 3$ of the twins taking each of these values.

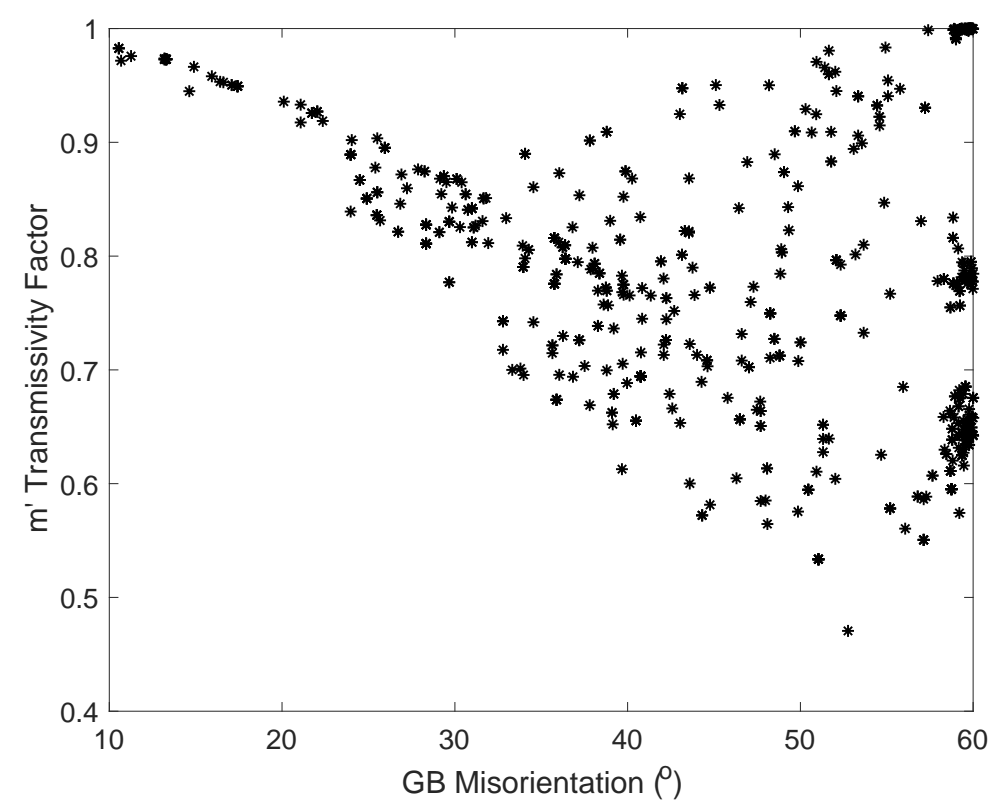

Figure 10. $M^{\prime}$ transmissivity factor vs misorientation for 660 slip bands.

The relationship between $\mathrm{m}^{\prime}$ factor and pileup stress is shown in Figure 11,and demonstrates a similar trend to GB misorientation - i.e. there is no significant correlation between $\mathrm{m}^{\prime}$ factor and pileup stress. The local average stress with increasing $\mathrm{m}^{\prime}$ factor is almost constant, as shown by the red diamonds in the figure; the outlying higher stress values that appear in the figure correlate with a higher density of points with a specific $\mathrm{m}^{\prime}$ value and are simply drawn from points further along the tails of the log-normal stress distribution as discussed previously. 


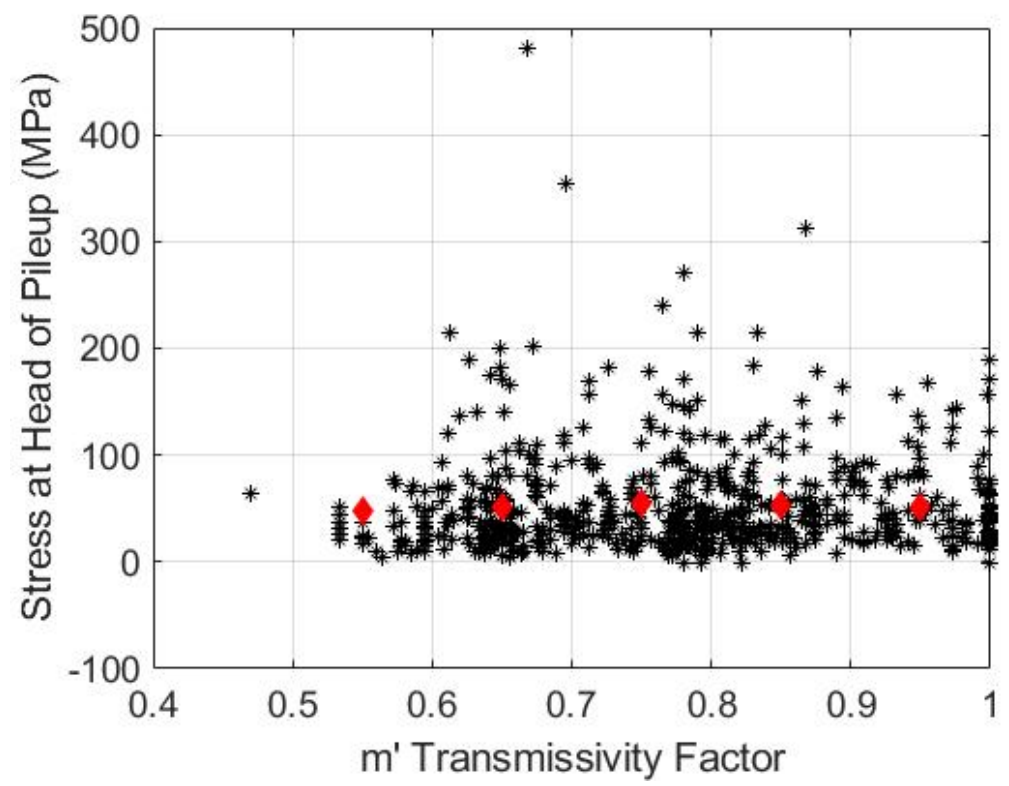

Figure 11. Pileup stress vs $m^{\prime}$ transmissivity factor for 660 slip bands in the RR1000 material (black asterisks); the mean value for a given $m^{\prime}$ range is shown as red diamonds.

All other transmissivity metrics considered here ( $\mathrm{N}$-factor, $\lambda$, residual Burgers vector) display the same independence from the pileup stress as mentioned earlier. Neither do the Schmid factors of the grain or its neighbor affect the pileup stress distribution. There is also no significant correlation between the angle of the GB trace and the pileup stress. However, there is a weak negative correlation between grain size and maximum stress at the head of the pileup ( $P$ value $>.005, R$-squared value of 0.0288 ).

The previous results, indicating weak or zero correlations between pileup stress and GB character are for the unloaded material; dislocations will certainly reorganize themselves to some extent during unloading. However, the observation of band transmission through a GB will still be apparent after unloading the sample.

Slip band transmission will now be qualitatively and quantitatively analyzed. It was found that $90 \%$ of the scanned sample surface demonstrated shear strain values of less than 0.06 at which point the top ten percent of shear values increased steeply to the maximum shear value in the scans. Therefore, 0.06 was taken to be assumed as within standard noise limits of no strain. Then through visually inspecting over 35 slip bands and their corresponding shear profiles, it was identified that transmitting slip bands demonstrated unique shear profile behavior at the GB compared to non-transmitting bands. Transmitting bands primarily had shear values above 0.06 at the GB while non-transmitting bands mostly showed the opposite. However, there was some overlap between shear values of about 0.06 and 0.07 ; therefore, bands with a shear value over 0.07 at the grain boundary were considered to be transmitting to ensure that there are no false positives of transmitted bands. Figure 12 shows an example shear profile of a transmitted 
band with non-zero shear strain at the GB. After applying this qualification to all 660 slip bands, it was found that roughly $35 \%$ of bands transmit through a grain boundary. Again, as mentioned earlier, transmission includes the concept of activation of a new slip system in a neighboring grain. Also it is important to note that in the sample, all slip bands that transmitted showed the ends of the slip bands that met at the GB were within one pixel of each other reinforcing the idea that in this sample transmission events often nucleate at or extremely close to the free surface.

After categorizing transmission events and revisiting the GB pileup stress, it was found that the average pileup stress for transmission events at $55 \mathrm{MPa}$ was roughly $45 \%$ higher than the average pileup stress of non-transmission events, which was $38 \mathrm{MPa}$. This could potentially be due to an increase in average stress required to transmit; however, there seems to be no indication of a consistent transmission threshold stress.

In situations where there was complete transmission and two discrete bands were identified on either side of the grain boundary, the bands were qualitatively observed. This qualitative observation of the resolved maximum shear strain profile provides insightful evidence of dislocation generation since local maximums would correlate with dominant dislocation generation sources as discussed previously. These shear strain profiles were observed from one end of the original slip band, along the band, across the grain boundary to the transmitted band and then on to the opposite end of the transmitted band. It was found that of these events, there were three cases of shear profiles that were observed in decreasing order as follows:

1. A profile containing one maximum found in one grain.

2. A profile containing two local maxima with one found in each grain.

3. A profile containing one maximum at the grain boundary 

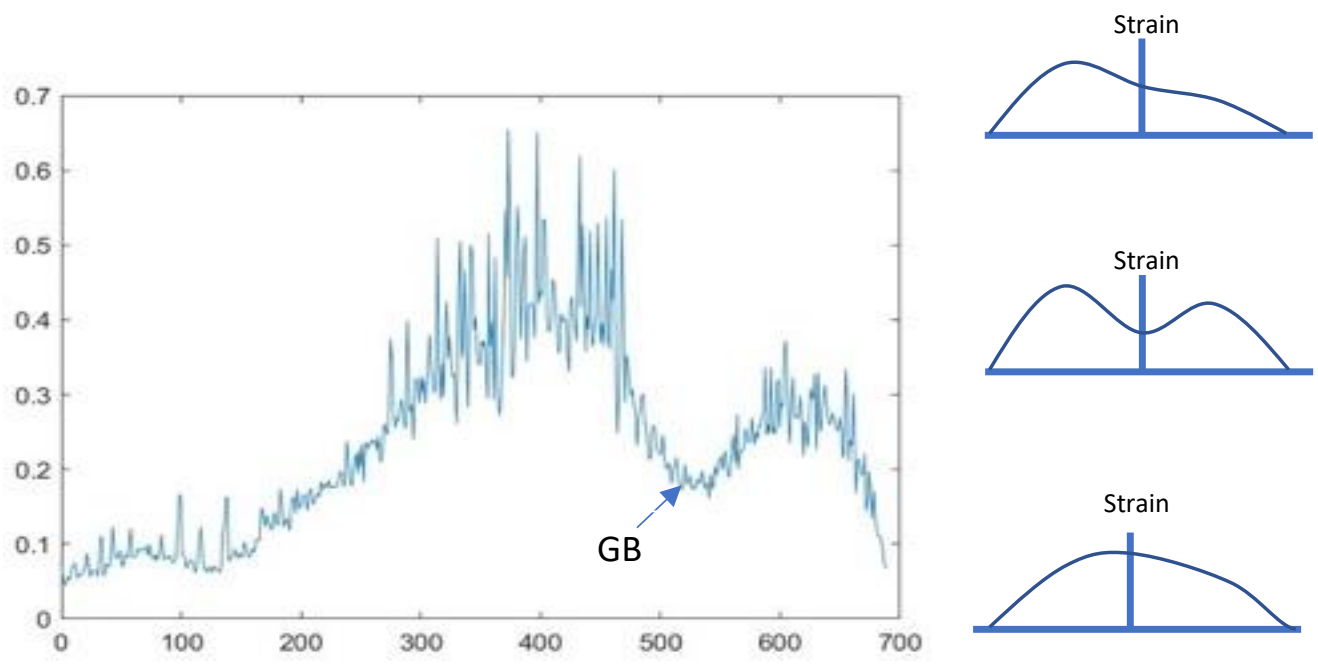

Figure 12. Shear profile of a slip band transmitted through a grain boundary with the local minimum correlating with the GB (left). Diagrams of primary dislocation generation in one grain (top right), both grains (middle right), and at the GB (bottom right). The vertical line indicates the GB.

Figure 12 gives an illustration of the three types of profiles with an example profile of the second case. As the maxima of these shear profiles correlate with locations of dislocation generation, this finding indicates where along the slip band the major dislocation sources lie. Case one suggests that there are instances in which the dislocations are generated in one grain and are transmitted through the grain boundary to the other grain. Case two suggests that there are instances in which dislocation generation is happening in both grains. While case three suggests that there are instances in which the dislocations are primarily generated at the grain boundary. Each one of these dislocation generation points would then correspond with primary Frank-Read dislocation sources for the slip band under the superdislocation model. 
We now look at quantitative GB character and its notable influence on slip band transmission. The relationship between transmitting events and GB misorientation is represented in Figure 13. Almost all bands with a low GB misorientation of below 20 degrees transmitted. This fraction of transmitting bands drops off steeply for the 20 to 30 degree GB misorientation set of bands and slowly increases as the GB misorientation increases toward that of a twin boundary where about $40 \%$ of the bands show transmission.

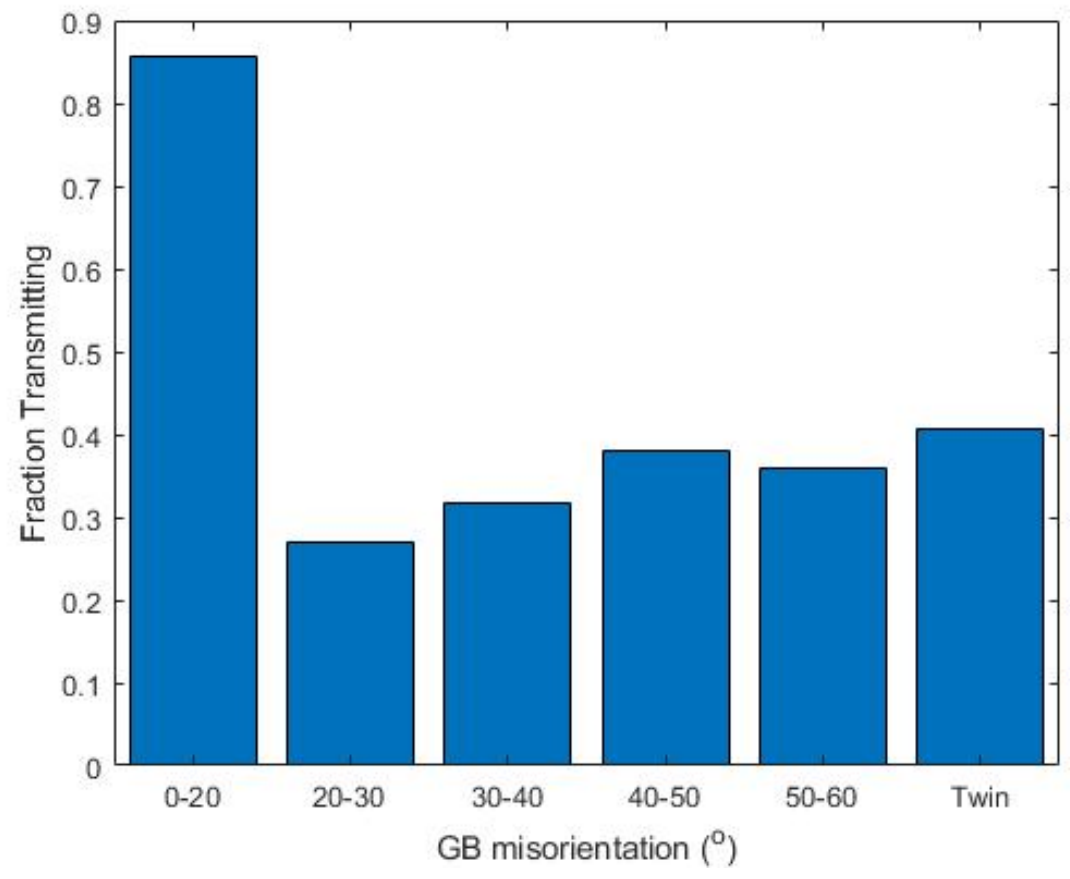

Figure 13. Fraction of transmitting slip bands divided by GB misorientation bins. Low misorientation relates to a high level of transmision.

Transmission events are also noticeabley influenced by $\mathrm{m}^{\prime}$ at high misorientation angles and twin boundaries. Figure 14 shows the relationship between the average $m^{\prime}$ value in discretized GB misorientation bins for transmitting and non-transmitting events. While there seems to be no distinguishable difference between the transmission and nontransmission events from 0 to 50 degrees, the $\mathrm{m}^{\prime}$ factor does influence transmission in higher misorientation levels of above 50 degrees. It would be expected that the average $\mathrm{m}^{\prime}$ transmissivity factor for low misorientation angles would be close to the same value for both transmission and non transmission events because the range of possible $\mathrm{m}^{\prime}$ transmissivity factor values decreases as GB misorentation angle decreases as was noted in Figure 10. 


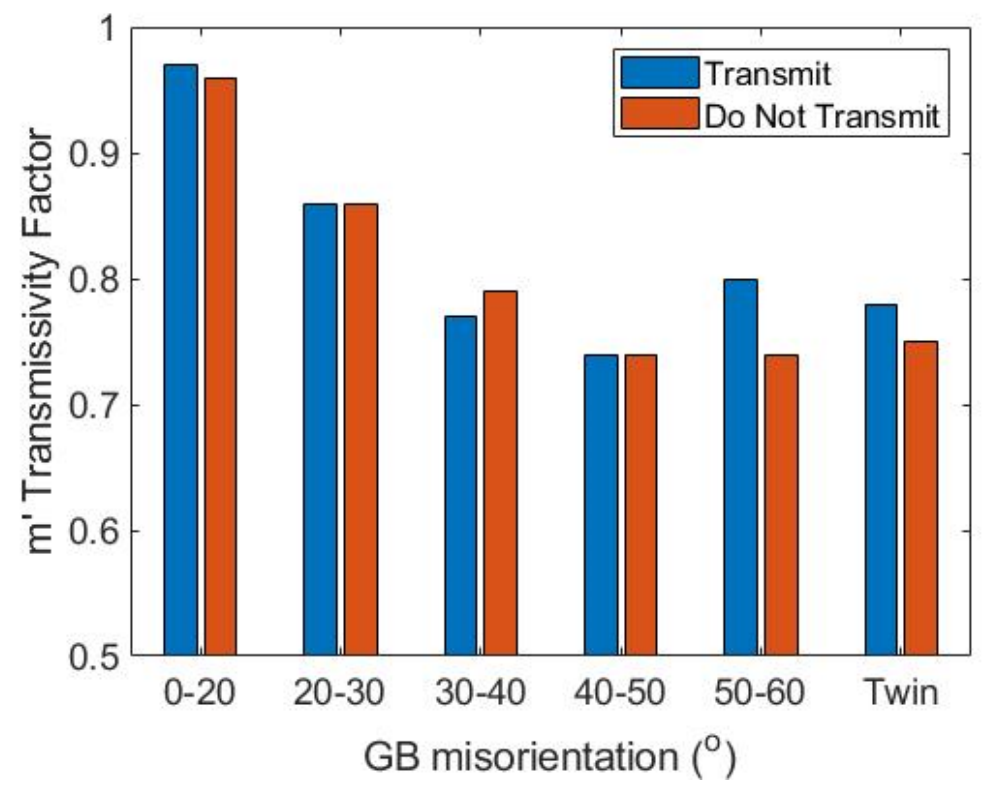

Figure 14. Average $m$ ' factor for bands that transmit and do not transmit divided into GB misorientation bins. Higher misorientations show a real but small difference in $m^{\prime}$ factor.

The distribution of $\mathrm{m}^{\prime}$ factors for transmission events at twin grain boundaries was then investigated. As was mentioned earlier, $\mathrm{m}^{\prime}$ factor values at twin grain boundaries assumed one of roughly three values which were .65, .78, and 1 (Figure 10). Respectively there were 104, 58, and 50 bands that had each of the three values. Figure 15 demonstrates the fraction of transmission and non transmission events for each of these three $\mathrm{m}^{\prime}$ factor values. As can be seen, the peak for transmission events lies at $\mathrm{m}^{\prime}$ factors of roughly 0.78 . Overall trends show that transmission increases with $\mathrm{m}^{\prime}$. However, these data suggest that at twin boundaries, GBs with an $\mathrm{m}^{\prime}$ factor of 1 have a surprisingly lower fraction transmitted than those with $\mathrm{m}^{\prime}$ of 0.78 . This could potentially be caused by elastic anisotropy and twin shape with parent grains causing unique impingements of slip which are different from that which is seen on other GB's. 


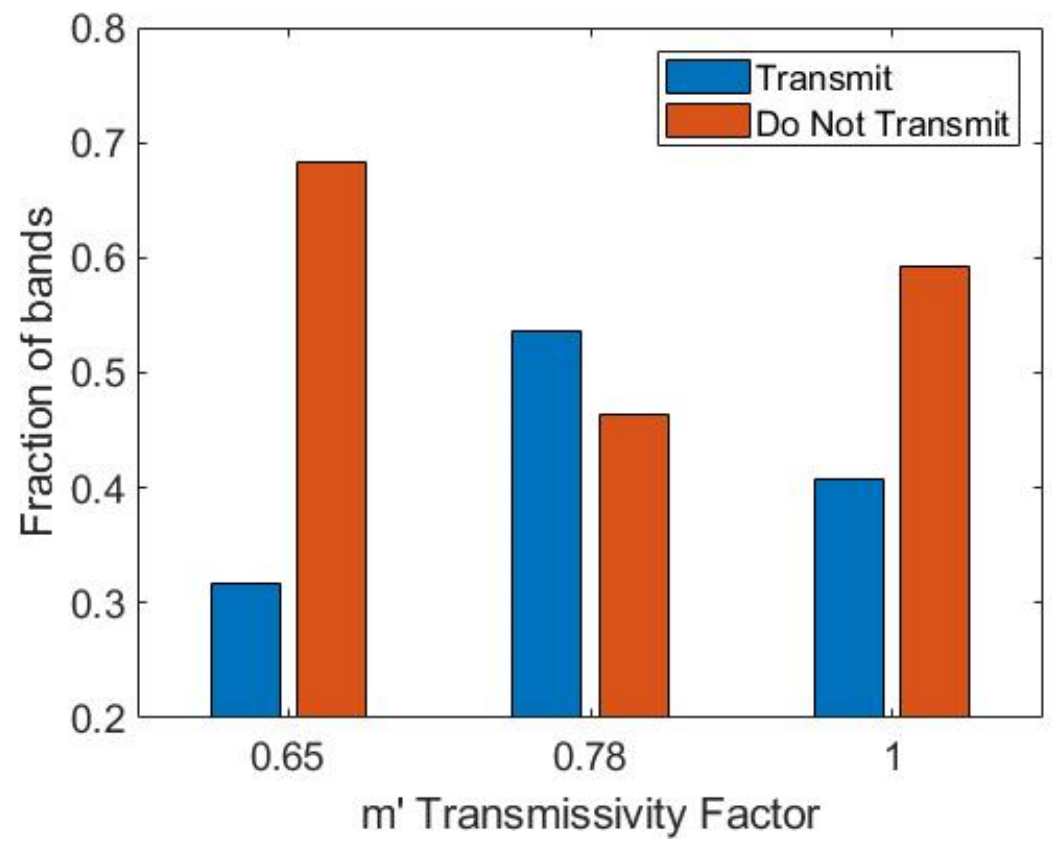

Figure 15. Fraction of bands that transmit at twin GB's divided into the three distinct $m$ ' factors found at twin GB's.

\section{Conclusion}

A surface applied gold speckled pattern enabled high-resolution strain data to be captured ( 117 nm per pixel) of the nickel-based super alloy. This allowed for the shear strain profiles along slip bands to be evaluated. It was noted that the shear strain profile along an 'average' band involved an increase in strain with distance from GB for approximately $1 / 3$ of the grain cross section, followed by a fairly flat region of relatively constant strain, with the strain dropping once again towards the opposite GB for the final $1 / 3$. The location of peak strain in the central $1 / 3$ of the cross section indicates that nucleation of most dislocations occurs towards the center $1 / 3$ of grains.

Based upon the observed strain gradients near GBs, GND spacing follows a similar profile to a single-ended pileup under applied stress, as analyzed by Eshelby and others.

The orientation gradient along a slip band dislocation pileup shows rotation about the line direction as identified by the RDR method. This is a new potential method to identify the active slip system using EBSD scanning alone that can be utilized in tandem with existing slip system identification methods. The direction of the rotation can be indicative of the dislocation sign observed at the surface. This follows characteristics of Frank-Read dislocation sources generating concetric dislocation loops that propagate outwards to either leave the sample surface or become pinned in a dislocation pileup. 
The distribution of pileup stresses at GBs, calculated by integrating elastic stresses between GNDs, follows a log-normal distribution that appears to be independent of GB character; i.e. the same stress distribution is present for GBs of all values of misorientation (greater than the minimum defined value of 10 degrees, and including twin GBs), and for all values of the transmissivity factors studied. It appears that all GBs with misorientation above 10 degrees have adequate obstacle strength to resist the pileup stress that the slip bands generate; i.e. the stress associated with the GNDs that form to ensure compatibility is lower than the obstacle strength of all GBs with misorientation greater than 10 degrees.

The pileup stress at the end of a slip band does correlate weakly with the maximum shear stress within a given grain; however, this maximum shear strain does not correlate with the Schmid factor of the grain (an observation that has been made by others).

Transmission is influenced by misorientation with transmission happening the most at low levels of misorientation. Additionally, at twin and high misorientation GBs transmission is also influenced by the $\mathrm{m}^{\prime}$ transmissivity factor. At twin GBs, the $\mathrm{m}^{\prime}$ value shows maximum transmission when $\mathrm{m}^{\prime}$ equals about 0.78. Transmission shear profiles show evidence of dislocation generation in both grains, the grain boundary, as well as generation in one grain with transmission to the other.

\section{Acknowledgements}

The BYU and OSU teams work was supported by U.S. Department of Energy (DOE), Office of Science, Basic Energy Sciences (BES), under Awards DE-SC0012587 and DE-SC00012483.

Fullwood also gratefully acknowledges the hospitality of the University of Manchester during the development of this work. Allan Harte would like to acknowledge EPSRC grant

EP/T012250/1 for time and resources. João Fonseca would like to aknowdged the LightForm EPSRC programme grant EP/R00171511. 


\section{References}

[1] M. Mrovec, C. Elsässer, P. Gumbsch, Interactions between lattice dislocations and twin boundaries in tungsten: A comparative atomistic simulation study, Philos. Mag. 89 (2009) 3179-3194. doi:10.1080/14786430903246346.

[2] D. V. Bachurin, D. Weygand, P. Gumbsch, Dislocation-grain boundary interaction in 〈1 1

1) textured thin metal films, Acta Mater. 58 (2010) 5232-5241. doi:10.1016/j.actamat.2010.05.037.

[3] H. Van Swygenhoven, P.M. Derlet, A.G. Frøseth, Nucleation and propagation of dislocations in nanocrystalline fcc metals, Acta Mater. 54 (2006) 1975-1983. doi:10.1016/j.actamat.2005.12.026.

[4] M.P. Dewald, W.A. Curtin, Multiscale modelling of dislocation/grain boundary interactions. II. Screw dislocations impinging on tilt boundaries in Al, Philos. Mag. 87 (2007) 4615-4641. doi:10.1080/14786430701297590.

[5] D.W. Adams, D.T. Fullwood, R.H. Wagoner, E.R. Homer, Atomistic survey of grain boundary-dislocation interactions in FCC nickel, Comput. Mater. Sci. 164 (2019) 171-185. doi:10.1016/j.commatsci.2019.04.007.

[6] J. Luster, M.A. Morris, Compatibility of deformation in two-phase Ti-Al alloys: Dependence on microstructure and orientation relationships, Metall. Mater. Trans. A. 26 (1995) 1745-1756. doi:10.1007/BF02670762.

[7] E. Werner, W. Prantl, Slip transfer across grain and phase boundaries, Acta Metall. Mater. 38 (1990) 533-537. doi:10.1016/0956-7151(90)90159-E.

[8] J.D. Livingston, B. Chalmers, Multiple slip in bicrystal deformation, Acta Metall. 5 (1957) 322-327. doi:10.1016/0001-6160(57)90044-5.

[9] A. Weidner, R. Beyer, C. Blochwitz, C. Holste, A. Schwab, W. Tirschler, Slip activity of persistent slip bands in polycrystalline nickel, Mater. Sci. Eng. A. 435-436 (2006) 540546. doi:10.1016/j.msea.2006.07.039.

[10] P.K. Madden, J.E. Harbottle, The imaging of slip bands in nickel, Phys. Status Solidi. 46 (1978) 639-652. doi:10.1002/pssa.2210460229.

[11] F. Di Gioacchino, J. Quinta da Fonseca, Plastic Strain Mapping with Sub-micron Resolution Using Digital Image Correlation, Exp. Mech. 53 (2013) 743-754. doi:10.1007/s11340-0129685-2.

[12] F. Di Gioacchino, J. Quinta Da Fonseca, An experimental study of the polycrystalline plasticity of austenitic stainless steel, Int. J. Plast. 74 (2015) 92-109. doi:10.1016/j.ijplas.2015.05.012.

[13] M.A. Tschopp, D.L. McDowell, Grain boundary dislocation sources in nanocrystalline copper, Scr. Mater. 58 (2008) 299-302. doi:10.1016/j.scriptamat.2007.10.010. 
[14] E.O. Hall, The deformation and ageing of mild steel: III Discussion of results, Proc. Phys. Soc. Sect. B. 64 (1951) 747-753. doi:10.1088/0370-1301/64/9/303.

[15] N.J. Petch, The Cleavage Strength of Polycrystals, J. Iron Steel Inst. 173 (1953) 25-28.

[16] Z. Shen, R.H. Wagoner, W.A.T. Clark, DISLOCATION PILE-UP AND GRAIN BOUNDARY INTERACTIONS IN 304 STAINLESS STEEL, Scr. Metall. 20(6) (1986) 921-926. doi:10.1016/0036-9748(86)90467-9.

[17] J.C.M. Li, Y.T. Chou, The role of dislocations in the flow stress grain size relationships, Metall. Mater. Trans. 1 (1970) 1145-1159. doi:10.1007/BF02900225.

[18] M.D. Sangid, T. Ezaz, H. Sehitoglu, I.M. Robertson, Energy of slip transmission and nucleation at grain boundaries, Acta Mater. 59 (2011) 283-296.

doi:10.1016/j.actamat.2010.09.032.

[19] Y. Guo, T.B. Britton, A.J. Wilkinson, Slip band-grain boundary interactions in commercialpurity titanium, Acta Mater. 76 (2014) 1-12. doi:10.1016/j.actamat.2014.05.015.

[20] S.J. Hessel, W. Voice, A.W. James, S.A. Blackham, C.J. Small, M.R. Winstone, Nickel alloy for turbine engine components, 6132527, 2000. doi:US005485919A.

[21] M.C. Hardy, B. Zirbel, G. Shen, R. Shankar, DEVELOPING DAMAGE TOLERANCE AND CREEP RESISTANCE IN A HIGH STRENGTH NICKEL ALLOY FOR DISC APPLICATIONS, 2004.

[22] A. Harte, A. Michael, M. Preuss, J.Q. Da Fonseca, On the measurement of deformation by electron backscatter diffraction and its suitability as an indicator of local plastic strain, (In Review)., 2019.

[23] J.K. Mackenzie, Second Paper on the Statistics Associated with the Random Disorientation of Cubes, Biometrika. (1958) 229.

[24] LaVision, Digital Image Correlation (DIC), DaVis 10. (n.d.). https://www.lavision.de/en/techniques/dic-dvc/index.php (accessed November 6, 2019).

[25] A. Harte, M. Atkinson, M. Preuss, J. Quinta da Fonseca, A statistical study of the relationship between plastic strain and lattice misorientation on the surface of a deformed Ni-base superalloy, (2020). doi:10.5281/ZENODO.3701817.

[26] J.M. Gere, B.J. Goodno, Mechanics of materials, Cengage Learning, 2013.

[27] Z. Chen, S.H. Daly, Active Slip System Identification in Polycrystalline Metals by Digital Image Correlation (DIC), Exp. Mech. 57 (2017) 115-127. doi:10.1007/s11340-016-0217-3.

[28] X. Xu, D. Lunt, R. Thomas, R.P. Babu, A. Harte, M. Atkinson, J.Q. da Fonseca, M. Preuss, Identification of active slip mode in a hexagonal material by correlative scanning electron microscopy, Acta Mater. 175 (2019) 376-393. doi:10.1016/j.actamat.2019.06.024.

[29] F.C. Frank, W.T. Read, Multiplication processes for slow moving dislocations [7], Phys. Rev. 79 (1950) 722-723. doi:10.1103/PhysRev.79.722. 
[30] Z.Q. Wang, I.J. Beyerlein, R. LeSar, Slip band formation and mobile dislocation density generation in high rate deformation of single fcc crystals, Philos. Mag. 88 (2008) 13211343. doi:10.1080/14786430802129833.

[31] J.D. Eshelby, F.C. Frank, F.R.N. Nabarro, The equilibrium of linear arrays of dislocations., London, Edinburgh, Dublin Philos. Mag. J. Sci. 42 (1951) 351-364. doi:10.1080/14786445108561060.

[32] M.F. Ashby, The deformation of plastically non-homogeneous materials, Philos. Mag. 21 (1970) 399-424. doi:10.1080/14786437008238426.

[33] D. Hull, D.J. Bacon, Introduction to Dislocations, 2011. doi:10.1016/C2009-0-64358-0.

[34] J.P. Hirth, J. Lothe, Theory of dislocations, Krieger Pub. Co, 1992.

[35] H. Lim, M.G. Lee, J.H. Kim, B.L. Adams, R.H. Wagoner, Simulation of polycrystal deformation with grain and grain boundary effects, Int. J. Plast. 27 (2011) 1328-1354. doi:10.1016/j.ijplas.2011.03.001.

[36] M.D. Atkinson, J.M. Donoghue, J.Q. Da Fonseca, Measurement of local plastic strain during uniaxial reversed loading of nickel alloy 625, Not yet Submitt. (n.d.) 124-155.

[37] M.J. Marcinkowski, W.F. Tseng, Dislocation behavior at tilt boundaries of infinite extent, Metall. Trans. 1 (1970) 3397-3401. doi:10.1007/BF03037870.

[38] M. Crimp, ECCl Analysis of Shear Accommodations at Grain Boundaries in Commercially Pure Alpha Titanium, Int. J. Plast. In Review (2019). 